\title{
Conductance of Aqueous Solutions of Lignosulfonate and Poly(styrene sulfonate)
}

\author{
Jean-Marie Hachey, Denise Maltais, Patricia Milani, \\ and Van Tam Bui* \\ Département des Sciences Fondamentales, Université du Québec à Chicoutimi, \\ Chicoutimi (Québec) Canada G7H 2 Bl \\ * Department of Chemistry and Chemical Engineering, Royal Military College, \\ Kingston (Ontario) Canada K7K 5 LO
}

(Received June 10, 1988)

\begin{abstract}
A procedure for isolating the high molecular-weight fraction of a commercial lignosulfonate sample by size exclusion chromatography is described together with the desalting process for that fraction. Conductance of aqueous solutions of the lithium, sodium, and potassium salts of the original lignosulfonate, the isolated lignosulfonate, and a standard poly(styrene sulfonate) sample were then measured at $25^{\circ} \mathrm{C}$. Experiments covered a large concentration range between 0.2 and about $100 \mathrm{eq} \mathrm{m}^{-3}$. The resulting equivalent conductance, for all cases, increased sharply when the concentration decreased to a very dilute limit after which it decreased steeply. Those results showing relatively high conductances for lignosulfonate allowed a comparison of its electrolyte behavior with that of poly(styrene sulfonate) on the basis of the complex chemical composition and structure of lignins.

KEY WORDS Conductance / Fractionation/Lignosulfonate / Poly(styrene sulfonate) / Size Exclusion Chromatography /
\end{abstract}

Lignin is one of major components of wood. It is usually isolated from the other main component, cellulose, by a sulfonation process converting it into lignosulfonates (LS) which are largely soluble in water. Although the exact structure of LS is very complex and also depends upon the kind of original wood as well as upon the sulfonation conditions, the overall structure of the LS molecule may be represented by Figure 1a.

The basic structure is a phenylpropane unit and the whole LS molecule may be represented as a wide variety of substituted aromatic chains cross-linked into a spiral form without definitive distribution. ${ }^{1,2}$ The sulfonate anions on the molecule surface, combined with the effect of the carboxylic and phenolic groups, make it easily soluble in water. In contrast with LS molecules, the chemical structure of poly(styrene sulfonate) (PSS), with the sul- fonate anions linked directly to the aromatic ring (moiety), appears to be much simpler; it doesn't contain any methoxyl, aldehydic, alcoholic, carboxylic or phenolic groups (see Figure 1b). Both molecules exhibit some interesting similarities: they are composed of one aromatic ring and one sulfonate group for every two or three $\mathrm{C}$ atoms in the lateral chains. This is one of the reasons why PSS polymers are currently used as reference standards in the study of lignosulfonates. However, because of many dissimilarities cited above, it is worth comparing the properties of LS and PSS polyions in aqueous media, without and with presence of single electrolyte like $\mathrm{NaCl}$. Electrical conductivity is commonly considered as one of the most representative dynamic properties of polyelectrolyte solutions. As the first step of our project, electrical conductivity measurements are performed on 


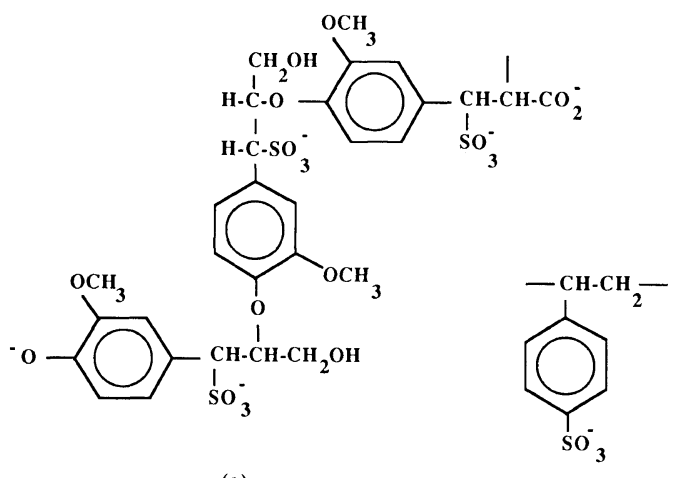

(a)

(b)

Figure 1. Illustrations of the chemical structures of lignosulfonate (a) and poly(styrene sulfonate) (b).

a salt-free solutions of these polyelectrolytes over a large concentration range.

\section{EXPERIMENTAL}

\section{Materials}

Poly(styrene sulfonate). The sodium poly(styrene sulfonate) sample was a standard one of molecular weight of $7 \times 10^{4}$ provided by Du Pont Instr. It had polydispersity index less than 1.10. The sample was passed through cation and anion exchange resins (Dowex types) repeatedly to remove all ionic impurities as well as to convert it into poly(styrene sulfonic acid) (HPSS), and finally freeze dried. HPSS aqueous solutions of various concentrations were then titrated with standard sodium hydroxide solutions, yielding the equivalent-weight of $187 \pm 2 \mathrm{~g} \mathrm{eq}^{-1}$ which is in good agreement with the theoretical value for $100 \%$ sulfonation. The lithium, sodium and potassium PSS salts were prepared by titration of HPSS solutions with appropriate hydroxides just to the equivalence point, and then isolated by freeze drying. The three hydroxides used were the purified grades obtained from Fisher Scientific.

Lignosulfonate. The sodium lignosulfonate sample was kindly given by Reed Company under the trade-name of Lignosol SFX-65. This product is sugar-free, high molecular weight sodium lignosulfonate (NaLS) prepared from sugar-free NaLS by ultrafiltration. The following elemental analysis data were provided by the supplier: $3.5 \%$ reductive materials, $7.5 \% \mathrm{Na}, 5.5 \%$ organic $\mathrm{S}$ and $6.3 \%$ total S. It can be deduced from these data that the ionic impurities content (in the form of $\mathrm{Na}_{2} \mathrm{SO}_{3}$ or $\mathrm{Na}_{2} \mathrm{SO}_{4}$ ) is about $0.8 \%$. The SFX-65 sample was purified and converted into $\mathrm{Li}^{+}, \mathrm{K}^{+}$salts by the same procedure as applied to PSS. Titrations of lignosulfonic acid (HLS) aqueous solutions of various concentrations yielded the equivalentweight of $258 \pm 3 \mathrm{geq}^{-1}$. This value is close to that predicted assuming $100 \%$ sulfonation which is $260 \mathrm{geq}^{-1}$ according to the basic structure of LS (Figure 1a).

Chromatography. Gel permeation chromatography (GPC) was performed for the LS sample. Due to the complexity of the chemical sulfonation process, the LS materials usually exhibit a polymodal pattern in their molecular weight distribution. Before embarking on any experiment, it is worth obtaining the pattern of the concerned LS sample. The GPC studies were performed on a glass column $(51 \times$ $1.7 \mathrm{~cm}$ ) packed with the Sephadex gel G-75. An aqueous $0.1 \mathrm{~N} \mathrm{NaCl}$ solution previously degassed was used as eluent. The concentration of injected LS aqueous solution was fixed at $0.2 \%$, with an injected volume of $100 \mu \mathrm{l}$. The flow rate of eluent was kept constant at $0.65 \mathrm{ml} / \mathrm{min}$. The UV detector was set at $280 \mathrm{~nm}$ being the optimum wavelength for LS molecules. Five standard poly(styrene sulfonate) samples (Du Pont Instruments Ltd.) with $\bar{M}_{w}$ varying from $4 \times 10^{3}$ to $7 \times 10^{4}$ $\bar{M}_{w} / \bar{M}_{n} \leq 1.10$, were used to calibrate the GPC system.

\section{Isolating and Desalting the High Molecular} Weight Fraction of the LS Sample

From the GPC chromatogram of the SFX65 sample presented in the next section, it is interesting and useful to isolate the high molecular weight (HMW) component and then 
perform conductivity measurements on it in order to compare it with the original material. The isolation was performed on a glass column $(18 \mathrm{~cm} \times 1.7 \mathrm{~cm})$ packed with $\mathrm{G}-75$ gel. The eluent used and its flow rate were the same as described above. An automatic injector introduced a fixed volume of a $5 \%$ LS solution into the column at periodic intervals, and the HMW fraction was collected by an automatic collector at predetermined periods of time. This operation lasted several hours. After isolation, the fraction was desalted using a PD-10 column packed with G-25 gel. This column was specifically prepared for this purpose by Pharmacia Company. The desalting operation needed a large amount of pure water as eluent flowing across the PD-10 column to clean out the $\mathrm{NaCl}$ in the $\mathrm{HMW}$ fraction. The effectiveness of this technique was checked by comparing the GPC results of that fraction recorded before and after desalting by means of a G-25 gel column monitored at the optimum wavelength of $210 \mathrm{~nm}$ which was considered suitable for the absorption of $\mathrm{NaCl}$.

\section{Conductance Measurement}

All the conductivity measurements were carried out at $25 \pm 0.02 \mathrm{C}$, by means of a digital conductometer from Yellow Spring Instruments, Model 32, with the electrodes lightly black-platinized. Polypropylene conductivity cells were preferred to the pyrex ones due to possible interference, specially at dilute concentrations, arising from ionic groups in the glass. The cell constant was determined by measuring the conductance of various concentrations of $\mathrm{KCl}$ solution whose specific conductances were provided by the apparatus supplier. The calculated cell constant was $100.3 \pm 0.2 \mathrm{~m}^{-1}$. This constant was checked intermittently during the periods of experiments. All conductivity measurements were made at a frequency of $3 \mathrm{kHz}$. It has already been observed ${ }^{3,4}$ that frequency has very little effect on the conductance results.

The LS or PSS solutions were prepared by weighing the previously vacuum dried samples in $50 \mathrm{ml}$ volumetric flasks which were filled up to the mark with freshly deionized-distilled water, after the material has dissolved in a little water. The resulting solutions were carefully protected from atmospheric $\mathrm{CO}_{2}$. The conductance of pure water, $K_{0}$, varied between 0.78 and $0.95 \times 10^{-4} \Omega^{-1} \mathrm{~m}^{-1}$ during a set of measurements.

In general, the uncertainty in the final results of equivalent conductance, $\Lambda$, for moderate and high concentration solutions was less than $1 \%$, being mainly due to errors in the concentration combined with those arising from the equivalent-weight determination. Very dilute solutions were obtained by diluting the more concentrated solutions in order to reduce errors in weighing. Experiments were repeated at least three times for each sample, the results were reproducible within an uncertainty of $2 \%$, due to a higher error in concentration determinations and a larger effect of the variation in pure water conductivity.

\section{RESULTS AND DISCUSSION}

\section{Molecular Weight Distribution and Isolation of the HMW Fraction}

Chromatograms of the SFX-65 sample, the HMW fraction and the PSS sample are presented together in Figure 2. They were all recorded on the same column, under the same experimental conditions. It was found that the original SFX-65 material was composed of a major narrow group of molecular weight of about 50,000 and a broader component whose peak was situated approximately at 5,000, all, according to a calibration curve obtained from five PSS standards. This is in good accordance with an average viscosimetric molecular weight of 31,500 previously observed for a similar LS sample. ${ }^{5}$ This type of GPC curve is usually encountered for lignosulfonate products. It represents the LS molecules formed from simultaneous depolymerization and repolymerization during the sulfonation process. $^{1}$ 


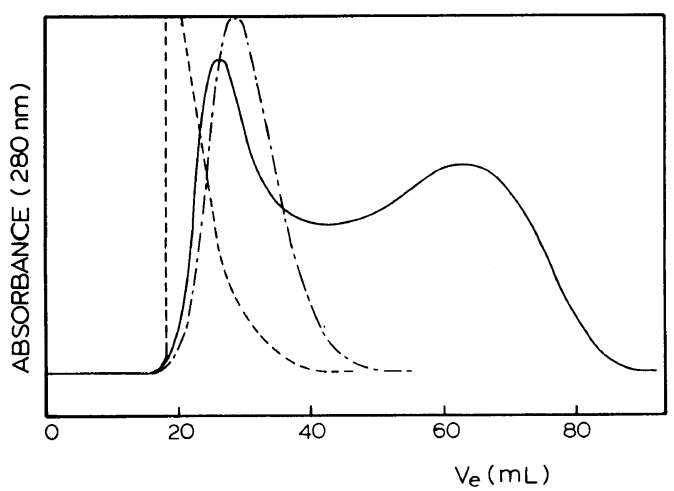

Figure 2. Molecular-weight distributions of: SFX-65 (full line), HMW (dotted line), and PSS (dashed line), using Sephadex G-75 gels.

Regarding the PSS sample and the HMW fraction, their molecular weight distributions are likely narrow as predicted, since the former is a standard one, and the latter obtained from fractionation. Finally, Figure 3, showing the chromatograms of the HMW fraction recorded before and after desalting indicates the effectiveness of the desalting process. The minor peak in this curve represents absorbance of $\mathrm{NaCl}$ at $210 \mathrm{~nm}$. It should be pointed out that the desalting process was essential because of the use of $0.1 \mathrm{~N} \mathrm{NaCl}$ aqueous solution as eluent in the preceding isolation process. The presence of $\mathrm{NaCl}$ in eluent was essential to suppress any interionic interactions between the gels and the LS molecules which might disturb the size exclusion performance in the column.

\section{Conductance Data for SFX-65 and HMW Samples}

The equivalent conductance, $\Lambda$, was obtained by dividing the difference between the measured conductivity, $K$, and that of pure water, $K_{0}$, by the equivalent concentration, $C$, of each solution. The latter was prealably computed using the equivalent-gramme value of $258 \mathrm{~g} \mathrm{eq}^{-1}$ for HLS as determined by titration. $\Lambda$ was then plotted against $\sqrt{C}$ in Figure 4. These curves bend upward pro-

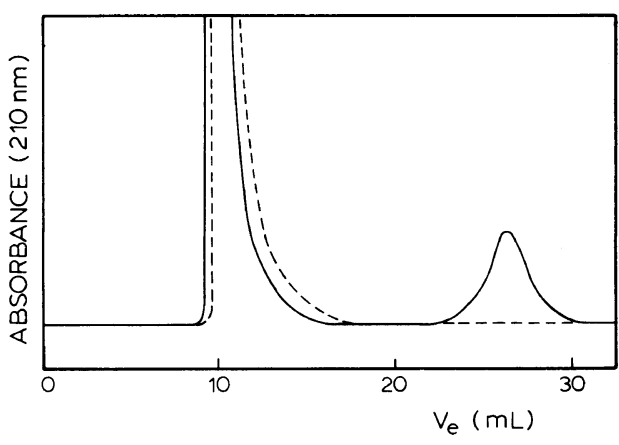

Figure 3. Molecular-weight profiles of the HMW fraction recorded before (full line) and after desalting (dashed line), using Sephadex G-25 gels.

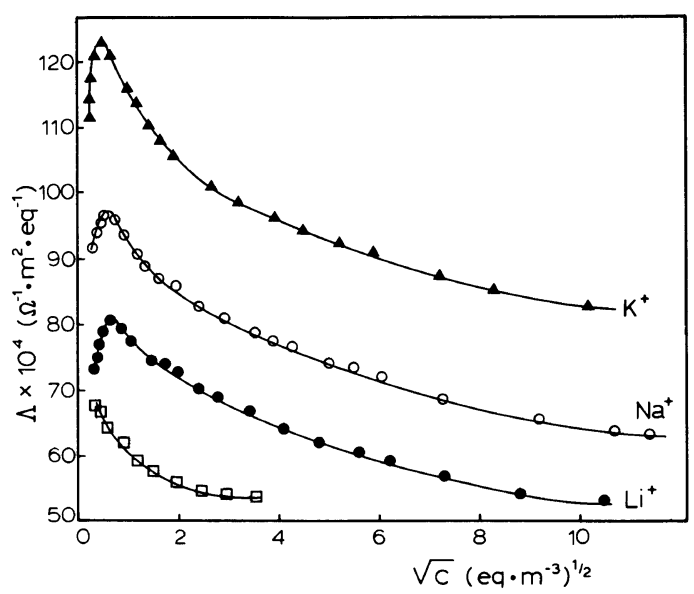

Figure 4. Concentration dependence of equivalent conductance for the SFX-65 and HMW samples ( $\square$, for HMW sample).

nouncedly as the concentration decreases. This kind of variation of $\Lambda$ for LS materials was first observed and interpreted by Gardon and Masson $^{6}$ who suggested lignosulfonates behave like expanding polyelectrolytes. However, in the very small concentration range, the curves bend downward very steeply due to the fact that $K_{0}$ is not constant but decreases in the presence of more and more polyelectrolyte since the fast $\mathrm{H}^{+}$ions in the counterion atmosphere are gradually replaced by slower ions like $\mathrm{Li}^{+}, \mathrm{Na}^{+}, \cdots$ so that the $\Lambda$ values obtained from $\left(K-K_{0}\right) / C$, with $K_{0}$ constant, should be more and more smaller than 
its real values in the very dilute region.

Regarding the HMW lignosulfonate fraction, measurements of conductivity were carried out from very low concentration until the limited solubility corresponding to approximately $1.5 \%$ weight/volume. The equivalent conductance results, calculated using a fixed value of $280 \mathrm{~g} \mathrm{eq}^{-1}$, are also reported in Figure 4 for the case of $\mathrm{Na}^{+}$cation. It is found that the conductance of the HMW fraction is much lower than that of the original SFX-65 sample. Therefore, it can be thought that the specific conductance of lignosulfonate polyion changes considerably with its molecular weight. This finding may be supported by the proposed ${ }^{1}$ change in structure between low and high molecular weight LS molecules.

\section{Conductance Data for the PSS Sample}

The equivalent conductances of NaPSS, LiPSS, and KPSS are all presented in Figure 5 which exhibit a sharp maximum in each curve and then deeply decreases at very low concentrations. Our results are very close to those reported $^{3}$ for a PSS sample of the same molecular weight, however, they are a little higher than those belonging to higher molecular weight samples, ${ }^{4.8}$ i.e., $2 \times 10^{5}$ and $5 \times 10^{5}$, respectively. The discrepancies may be attributed to differences in the origins of the samples used.

\section{Comparison between LS and PSS Conduct- ances}

The equivalent conductance of the PSS sample is much lower than that of the SFX65 sample. This important difference may be mainly assigned to the large effect of the low molecular weight component in the LS material rather than to some abnormal difference between specific conductances of the LS and PSS polyions. This finding is also supported by a smaller difference between the results of the HMW fraction and the PSS sample.

The Manning's theory, ${ }^{9}$ for expressing the equivalent conductance at a moderate or high

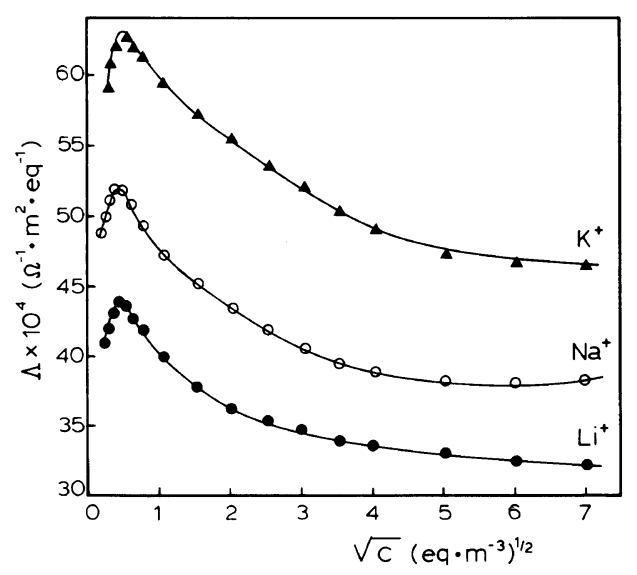

Figure 5. Concentration dependence of equivalent conductance for the PSS sample.

concentrations can be expanded as follows:

$$
\Lambda=f\left(\lambda_{\mathrm{p}}+\lambda_{\mathrm{c}}^{\circ}\right)
$$

Here $f$ and $\lambda_{\mathrm{p}}$ are interdependent and they depend obviously on the nature of polyion as well as on the type of counterion. Nevertheless, for the purpose of comparison, we attempted to use the results for any pair of counterions for a rough estimation of $f$ and $\lambda_{\mathrm{p}}$ for some typical concentrations. The eq 1 was solved using the available values ${ }^{10}$ of $\lambda_{\mathrm{c}}^{\circ}: 38.7$, 50.1, and $73.5 \Omega^{-1} \mathrm{~m}^{2}$ for $\mathrm{Li}^{+}, \mathrm{Na}^{+}$, and $\mathrm{K}^{+}$, respectively. The values listed in Table I were obtained by using the pair of $\mathrm{Na}^{+}$and $\mathrm{K}^{+}$for both samples.

These results reveal two interesting facts. First, the interaction parameter $f$ for LS material is much higher than PSS material;. secondly, the specific conductivity, $\lambda_{p}$, of LS polyion is slightly larger than that of PSS polyion. These differences may be regarded as consequences of the following causes. Firstly, LS molecule should have a spherical compact shape due to cross-links and to complexity of its chemical composition, while the PSS molecule behaves as a rod-like chain in aqueous solutions. Secondly, the presence of many carboxylic and phenolic groups in each LS molecule must contribute to some extent to 
Table I. $f$ and $\lambda_{\mathrm{p}}$ values for both SFX-65 and PSS samples at some moderate and high concentrations

\begin{tabular}{|c|c|c|c|c|c|}
\hline \multirow{2}{*}{ Sample } & $C$ & $10^{4} \Lambda_{\mathrm{Nap}}$ & $10^{4} \Lambda_{\mathrm{Kp}}$ & \multirow{2}{*}{$f$} & $\lambda_{\mathrm{p}}$ \\
\hline & eq $\mathrm{m}^{-3}$ & $\Omega^{-1} \mathrm{~m}^{2} \mathrm{eq}^{-1}$ & $\Omega^{-1} \mathrm{~m}^{2} \mathrm{eq}^{-1}$ & & $\Omega^{-1} \mathrm{~m}^{2} \mathrm{eq}^{-1}$ \\
\hline \multirow{3}{*}{$\begin{array}{l}\text { SFX-65 } \\
\text { (original) }\end{array}$} & 1.0 & 88.0 & 108.5 & 0.88 & 50.3 \\
\hline & 4.0 & 80.2 & 99.5 & 0.83 & 45.8 \\
\hline & 9.0 & 75.5 & 94.5 & 0.81 & 42.9 \\
\hline \multirow[t]{3}{*}{ PSS } & 1.0 & 47.3 & 59.7 & 0.54 & 38.5 \\
\hline & 4.0 & 43.5 & 55.5 & 0.51 & 34.7 \\
\hline & 9.0 & 40.8 & 52.2 & 0.49 & 33.6 \\
\hline
\end{tabular}

its conductivity. Furthermore, the sulfonate groups are directly attached to the main propyl units in LS molecules while they are fixed to the aromatic rings in the PSS molecules. Finally, as already mentioned, the polydispersity of LS material can give rise to an appreciable effect on its conductivity. In further works, it would be determined if the presence of various amounts of $\mathrm{NaCl}$ in the $\mathrm{LS}$ and PSS solutions could attenuate the differences between their electrolytic properties.

\section{CONCLUSION}

The high molecular weight fraction of commercial lignosulfonates has an electrical conductivity higher than that of poly(styrene sulfonate) of the nearly same molecular weight. This difference may be likely attributed to the complexities existing in the chemical composition and in the shape of lignosulfonate molecules. Meanwhile, the LS commercial sample SFX-65 presents a conductivity well above that of PSS sample; this cartainly arises from the presence of low molecular weight LS molecules whose electrolytic behavior should not be the same like polyelectrolytes.

\section{REFERENCES}

1. K. Forss and K. E. Fremer, Int. Symp. Wood Pulping Chemistry, Vol. 4, Ekman-Days, 1981, pp 29-38.

2. J.-M. Hachey, V. T. Bui, Y. Tremblay, D. Houde, and M. Mihelich, J. Wood Chem. Technol., 6, 389 (1986).

3. J. Szymczak, P. Holyk, and P. Ander, J. Phys. Chem., 79, 269 (1975).

4. D. Dolar, J. Span, and A. Pretna, J. Polym. Sci., C, 16, 3557 (1968).

5. V. T. Bui, J.-M. Hachey, and Y. Tremblay, Can. J. Chem. Eng., 64, 517 (1986).

6. J. L. Gardon and S. G. Mason, Can. J. Chem., 33, 1477 (1955).

7. H. Vink, J. Chem. Soc., Faraday Trans. 1, 77, 2439 (1981).

8. J. C. T. Kwak and R. C. Hayes, J. Phys. Chem., 79, 265 (1975).

9. G. S. Manning, J. Phys. Chem., 79, 262 (1975).

10. R. A. Robinson and R. H. Stokes, "Electrolytic Solutions," 2nd ed, Butterworths, London, 1959. 